16

\title{
Кинетика затухания теплового излучения поверхностных слоев углеродных материалов при импульсном лазерном возбуждении
}

\author{
(C) C.E. Зеленский ${ }^{1}$, T. Аоки² \\ ${ }^{1}$ Киевский национальный университет имени Тараса Шевченко, \\ 01601 Киев, Украина \\ ${ }^{2}$ Исследовательский институт электроники, Университет Шизуоки, \\ 432-8011Хамамацу, Япония \\ e-mail: zele@univ.kiev.ua
}

Поступила в редакцию 19.06.2019 г.

В окончательной редакции 19.06.2019 г.

Принята к публикации 08.07.2019 г.

Экспериментально и теоретически изучено затухание теплового излучения поверхностных слоев углеродных материалов при возбуждении импульсами неодимового лазера с модуляцией добротности. Обнаружено, что кривые затухания можно с удовлетворительной точностью аппроксимировать суммой двух экспоненциальных компонент с временами затухания порядка 10 и $100 \mathrm{~ns}$. При облучении образцов последовательностью лазерных импульсов наблюдаемые изменения кривых затухания свечения можно представить как перераспределение интенсивности вышеупомянутых двух компонент. По результатам компьютерного моделирования сделан вывод, что время затухания свечения определяется отношением глубины проникновения лазерного излучения к длине тепловой диффузии, что открывает возможность для определения коэффициента температуропроводности исследуемого материала в тонком поверхностном слое при высоких температурах (тысячи Кельвинов).

Ключевые слова: импульсное лазерное возбуждение, тепловое излучение, углеродные материалы, температуропроводность.

DOI: $10.21883 /$ OS.2019.11.48528.217-19

\section{Введение}

При импульсном лазерном возбуждении конденсированных сред анализ вторичного излучения (люминесценции, рассеяния) является широко распространенным направлением в спектроскопии и дает важную информацию о свойствах облучаемого материала и о процессах взаимодействия излучения с веществом. В отличие от люминесценции и рассеянного света индуцированное лазером тепловое излучение изучено менее подробно, и вопрос об информативности этого типа вторичного излучения далек от окончательного решения и остается предметом исследований.

Известно, что при локальном лазерном нагреве материала возникают разнообразные процессы, для которых характерным является сосуществование трех типов волн: оптических, температурных и акустических $[1,2]$. К настоящему времени разработаны и нашли применение экспериментальные методы, использующие лазерный нагрев материалов и позволяющие получать важную информацию об их оптических, тепловых и механических (акустических) свойствах. Важное место среди упомянутых занимают методы, использующие тепловое излучение, возникающие при лазерном нагреве материалов.

Например, в работах [3-5] рассматривается разновидность метода инфракрасной эмиссионной спектроскопии - метод индуцированного лазером теплового излучения (LITE, laser-induced thermal emission), в котором исследуемый материал нагревается лазерным излучением, и спектр теплового излучения содержит информацию о химическом составе материала. Следует также упомянуть группу методов под общим названием фототермическая радиометрия (PTR, photo-thermal radiometry) [6-15], в которых оптическое (лазерное) излучение создает относительно небольшой (порядка десятков градусов) локальный нагрев исследуемого материала, и это изменение температуры фиксируется по тепловому излучению в инфракрасной области спектра. B методах PTR обычно используют импульсные или модулированные лазерные источники (соответственно речь идет о методах импульсной или модулированной PTR). Например, в литературе рассматриваются применения PTR для измерения параметров теплопроводности материалов [8-11], для измерения температуры образцов [13-15].

В упомянутых выше методах в тех частях исследуемого материала, в которых происходит поглощение лазерного излучения, температура обычно повышается на несколько десятков градусов, и это изменение температуры фиксируется по тепловому излучению в инфракрасной области спектра. При этом средняя температура исследуемого материала (в отсутствие лазерного облучения) может варьироваться и обычно не превышает $1000 \mathrm{~K}$.

Особый интерес представляет случай, когда лазерное излучение нагревает облучаемый участок исследуемого материала до температур в несколько тысяч градусов. 
Такие условия легко достигаются на практике, если лазерный нагрев локальный и кратковременный. Локальность нагрева можно обеспечить за счет высокого коэффициента поглощения на длине волны лазерного излучения (например, $10^{6}-10^{7} \mathrm{~m}^{-1}$ ), а кратковременность нагрева обеспечивается применением достаточно коротких лазерных импульсов (например, наносекундные лазерные импульсы с поверхностной плотностью энергии порядка $0.1-1 \mathrm{~J} \cdot \mathrm{cm}^{-2}$ ). В таких случаях индуцированное лазером тепловое излучение имеет значительный вклад в видимом диапазоне спектра и может быть замечено даже невооруженным глазом. Данный режим лазерного нагрева открывает дополнительные возможности для исследования свойств материалов при высоких температурах, при которых применение традиционных методов затруднительно.

Например, среди высокотемпературных методов, использующих индуцированное лазером тепловое излучение, следует отметить методы LII (laser-induced incandescence) для углеродных микрочастиц в аэрозолях, которые используются для мониторинга процессов горения [16-22]. Что касается конденсированных сред, то видимое тепловое излучение светопоглощающих микрочастиц, взвешенных в прозрачных жидкостях, полимеpax, стеклах, исследовалось в работах [23-29] при возбуждении импульсами неодимового лазера с модуляцией добротности. Было замечено, что данный тип вторичного излучения характеризуется нелинейной зависимостью от интенсивности лазерного возбуждения и сопровождается образованием микроскопических пузырьков в окрестности микрочастиц, что приводит к существенным лазерно-индуцированным изменениям светорассеяния в суспензиях и оптическому ограничению [30-34].

В непрозрачных (сильно поглощающих) средах индуцированное лазером тепловое излучение в видимом диапазоне исследовалось в работах [35-42]. Данное свечение наблюдалось в углеродных материалах и в некоторых полупроводниках. Из особенностей данного типа свечения следует отметить его сильную нелинейность и зависимость от предыстории лазерного облучения. Кроме того, на микроскопическом уровне светимость облучаемой поверхности оказывается в значительной степени неоднородной из-за наличия микроструктуры или шероховатостей.

Настоящая работа посвящена изучению особенностей кинетики затухания индуцированного лазером видимого теплового излучения поверхностных слоев сильно поглощающих материалов (на примере углеродных материалов) при импульсном лазерном возбуждении. Как оказалось, при выполнении определенного соотношения между тепловыми и оптическими характеристиками исследуемого материала в затухании индуцированного лазером теплового излучения могут наблюдаться две стадии с характерными временами, отличающимися на порядок (например, $10^{-8}$ и $10^{-7} \mathrm{~s}$ ), причем величина времени затухания на „медленной“ стадии легко определяется из эксперимента и несет информацию о коэффициентах поглощения и температуропроводности поверхностного слоя исследуемого материала.

\section{Методы}

В работе использовалось излучение ИАГ: $\mathrm{Nd}^{3+}$-лазера с модуляцией добротности (длина волны $1064 \mathrm{~nm}$, длительность импульса $20 \mathrm{~ns}$ ). Лазер работал в режиме одиночных импульсов; поверхностная плотность мощности составляла от 15 до $30 \mathrm{MW} \cdot \mathrm{cm}^{-2}$ для образцов разных типов. При измерениях образцы облучались последовательностью одинаковых лазерных импульсов с интервалом между импульсами $50 \mathrm{~s}$. При этом возникали импульсы свечения, которые регистрировались (каждый импульс отдельно) фотоумножителем Н1949-51 (время нарастания $1.3 \mathrm{~ns}$ ) через стеклянный светофильтр СС-4 (область пропускания 400-500 nm) цифровым осциллографом TDS-2022B (полоса пропускания $200 \mathrm{MHz}$ ). Поскольку в процессе облучения последовательностью лазерных импульсов образец не сдвигался, то номер лазерного импульса в последовательности (далее в работе обозначается $N$ ) является также мерой дозы лазерного облучения поверхности образца.

Основная часть измерений была проведена с использованием образцов двух типов, которые изготавливались из угольных электродов для спектроскопии. У образцов типа А поверхность, предназначенная для лазерного облучения, предварительно шлифовалась и затем полировалась на плоской поверхности, покрытой листом бумаги, без абразива до получения металлического блеска (контролировалось визуально). У образцов типа В облучаемая поверхность образовывалась путем скола углеродного стержня без каких-либо дополнительных обработок. Типичные СЭМ-изображения поверхностей образцов А и В приведены на рис. 1.

Угольные электроды, использовавшиеся для изготовления образцов, хранились при комнатной температуре и относительной влажности около 30-40\%. Лазерное облучение и измерения проводились при той же температуре и влажности сразу после изготовления образцов.

\section{Результаты и их обсуждение}

\section{Эксперимент}

Типичные осциллограммы индуцированного лазером теплового излучения полированных (А) и сколотых (В) поверхностей приведены на рис. 2. Цифры возле кривых представляют собой номера $N$ импульсов в последовательности лазерного облучения. Интенсивность лазерного возбуждения для образцов А и В составляла 15 и $30 \mathrm{MW} \cdot \mathrm{cm}^{-2}$ соответственно. Приведенные на рис. 2 осциллограммы нормированы по максимуму.

Как видно из рис. 2, кинетика затухания свечения отличается у образцов разных типов и, кроме того, зависит от предшествующего лазерного облучения поверхности образцов. Так, у образцов типа А влияние лазерного облучения относительно невелико: осциллограммы $N=5$ и 40 практически совпадают, а осциллограмма $N=1$ отличается от них незначительно. С другой стороны, у 

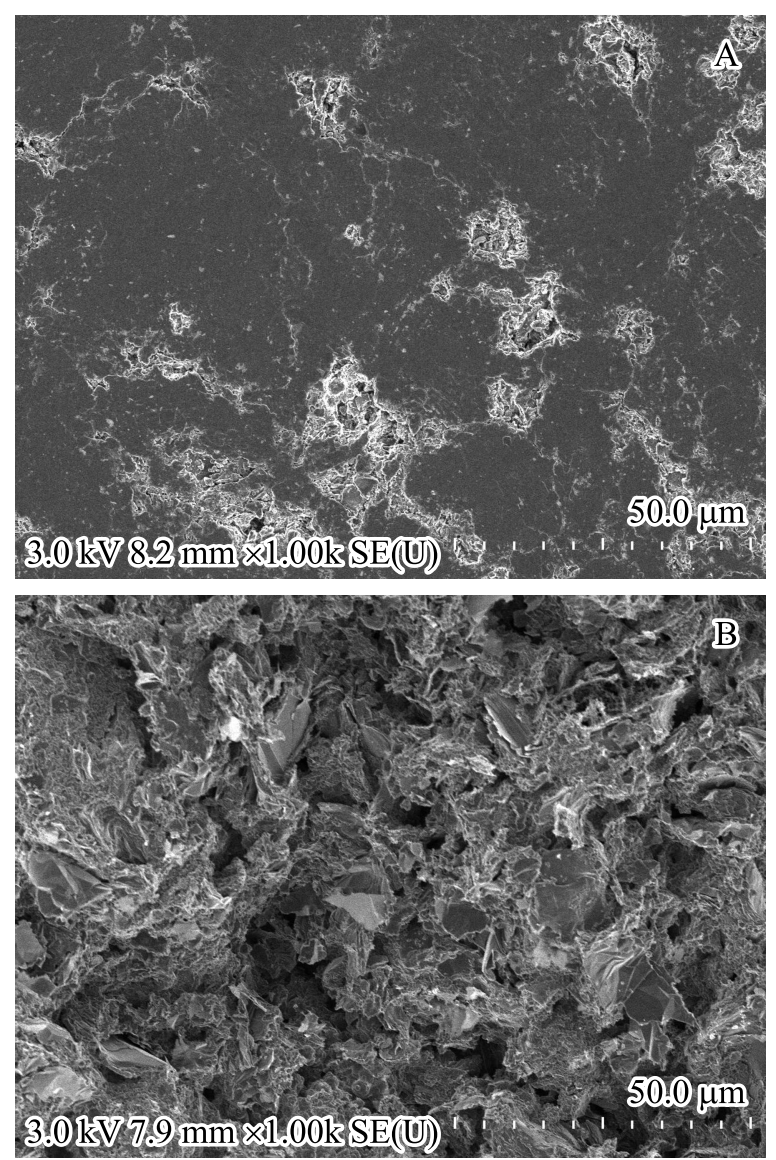

Рис. 1. СЭМ-изображения поверхностей исследуемых образцов типа А и В.

образцов типа В лазерное облучение от $N=1$ до $N=40$ приводит к заметному сокращению импульсов свечения.

Следует отметить, что форму кривой затухания теплового излучения при импульсном лазерном возбуждении можно определить решением уравнения теплопроводности с использованием формулы Планка для излучения черного тела, однако аналитическое выражение для кривой затухания свечения в рассматриваемом нами случае остается неизвестным. Попытки аппроксимировать имеющиеся экспериментальные данные экспоненциальной функцией $I=I_{0} e^{-t / \tau}$ оказываются неудачными (см., например, штриховую кривую на рис. 3), в то время как использование выражения

$$
I=I_{1} \exp \left(-t / \tau_{1}\right)+I_{2} \exp \left(-t / \tau_{2}\right)
$$

с подгоночными параметрами $I_{1}, I_{2}, \tau_{1}, \tau_{2}$ дает вполне удовлетворительные результаты (например, сплошная кривая на рис. 3) для всех имеющихся осциллограмм. Хотя выражение (1) и не имеет строгого теоретического обоснования, в настоящей работе оно дает простой и удобный путь для сравнения кривых затухания, полученных на разных образцах при разных значениях $N$.

Как показано в работах $[35,38,40]$, при облучении углеродных материалов последовательностью лазерных импульсов в облучаемых поверхностных слоях происходят трансформации, которые приводят к зависимости интегральной интенсивности свечения от дозы лазерного облучения $N$. Влияние лазерного облучения на кинетику затухания теплового излучения в предыдущих работах не рассматривалось. В настоящей работе обнаружено, что параметры кривых затухания $I_{1}, I_{2}, \tau_{1}$, $\tau_{2}$ существенно изменяются при наборе дозы лазерного облучения как для полированных, так и для сколотых поверхностей. Как видно из рис. 4, в затухании свечения можно выделить две компоненты, у которых времена затухания $\left(\tau_{1}\right.$ и $\left.\tau_{2}\right)$ при наборе дозы лазерного облучения изменяются слабо, в то время как отношение их интенсивностей $I_{1} / I_{2}$ возрастает примерно вдвое с ростом $N$. Следует отметить, что ниже в настоящей работе величина времени затухания быстрой компоненты $\tau_{1}$ не анализируется, поскольку она сравнима с длительностью заднего фронта лазерного импульса,

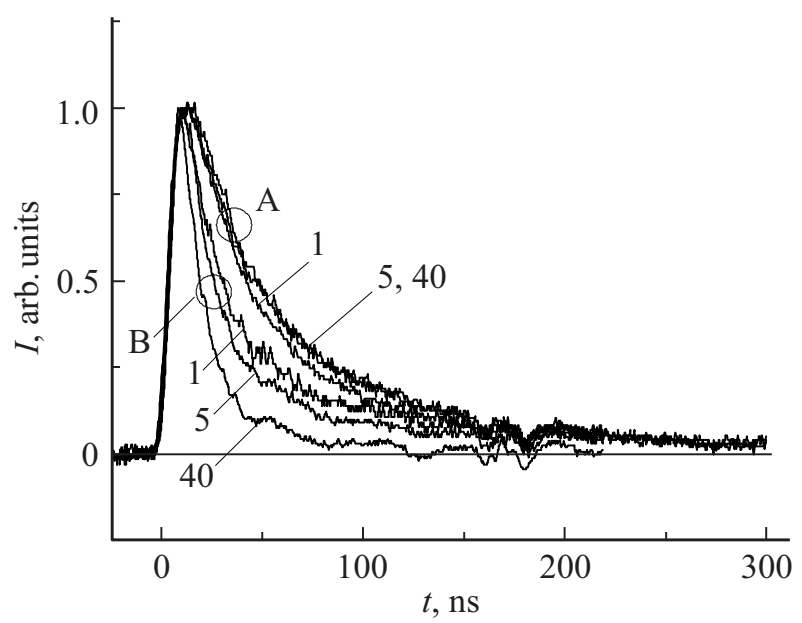

Рис. 2. Типичные нормированные осциллограммы полированных (А) и сколотых (В) поверхностей при облучении последовательностью лазерных импульсов. Цифры возле кривых номера импульсов в последовательности.

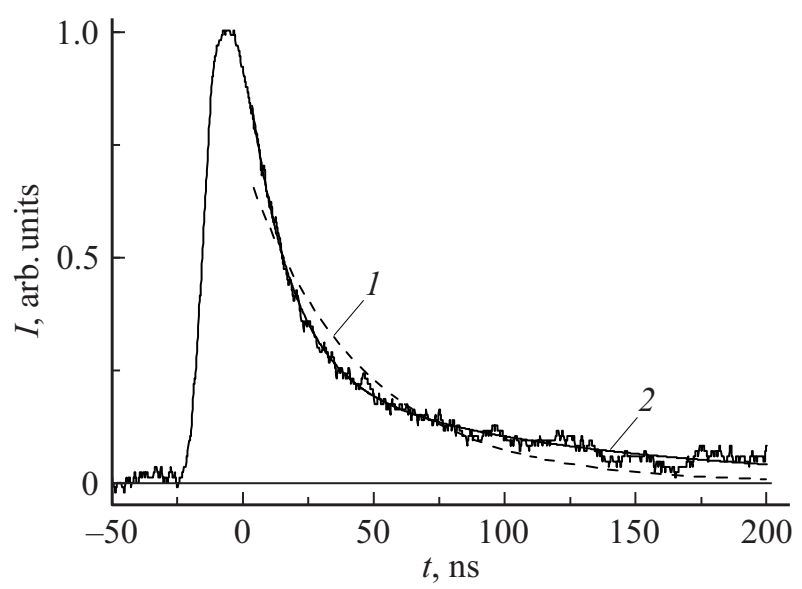

Рис. 3. Аппроксимация кривой затухания свечения образца типа В, $N=1$, выражением $I=I_{0} e^{-t / \tau}$ (кривая 1 ) и выражением (1) (кривая 2). 

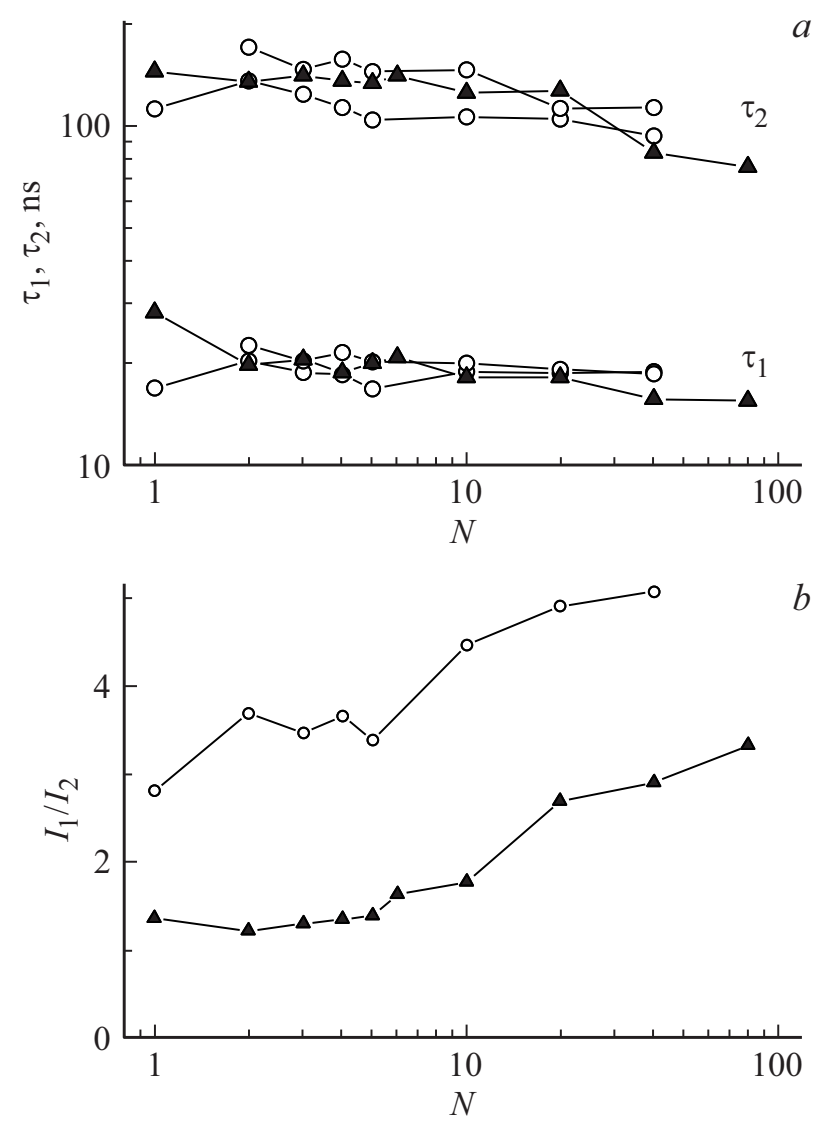

Рис. 4. Параметры $\tau_{1}, \tau_{2}(a)$ и $I_{1} / I_{2}(b)$ кривых затухания свечения как функции дозы лазерного облучения $N$ для образцов типа А (треугольники) и В (кружки).

что не дает возможности определить $\tau_{1}$ по результатам измерений с удовлетворительной точностью.

\section{Компьютерное моделирование}

Лазерный нагрев поверхностного слоя светопоглощающего материала можно смоделировать с использованием классического уравнения теплопроводности

$$
\operatorname{div}(\kappa \operatorname{grad}(T))+\alpha F=\rho C_{p} \frac{\partial T}{\partial t},
$$

где $\kappa$ - коэффициент теплопроводности, $T=T(\mathbf{r}, t)-$ температура в точке с координатами $\mathbf{r}$ в момент времени $t, \alpha-$ коэффициент поглощения на длине волны лазерного излучения, $F-$ интенсивность (поверхностная плотность мощности) лазерного излучения, $C_{p}$ и $\rho$ - соответственно удельная теплоемкость и плотность материала. Предполагается, что перечисленные параметры материала не зависят от интенсивности лазерного излучения и температуры.

Для зависимости лазерной интенсивности от времени в расчетах использовались приближения прямоугольного и гауссова импульсов с длительностью $\tau_{i}=20 \mathrm{~ns}$.

В настоящей работе важным для анализа параметром является длина тепловой диффузии, определяемая выра- жением

$$
\delta=\sqrt{D \tau_{i}}
$$

где $D=\kappa\left(C_{p} \rho\right)^{-1}-$ коэффициент температуропроводности.

Распространение лазерного излучения в облучаемом материале можно представить следующим уравнением:

$$
d F / F=-\alpha d z,
$$

где ось $z$ совпадает с направлением распространения лазерного излучения и перпендикулярна облучаемой поверхности образца. Для упрощения полагается, что $F$ не зависит от координат $x$ и $y$.

Введем глубину проникновения лазерного излучения в облучаемый материал следующим выражением:

$$
\Delta=\alpha^{-1}
$$

Для расчета светимости поверхности $\varepsilon$ в узком спектральном интервале $\Delta \lambda$ используем формулу Планка для абсолютно черного тела

$$
\varepsilon=\frac{2 \pi h c^{2} \lambda^{-5} \Delta \lambda}{\exp (h c / \lambda k T)-1},
$$

где $h-$ постоянная Планка, $c-$ скорость света, $k-$ постоянная Больцмана.

Для расчета светимости $\varepsilon$ как функции времени в выражение (4) подставляется решение $T=T(\mathbf{r}, t)$ уравнений (2) и (3) при $z=0$ (поверхность образца).

В работе [39] рассматривалось влияние коэффициента теплопроводности материала на кинетику теплового излучения при импульсном лазерном возбуждении при условии, что глубина проникновения лазерного излучения меньше длины тепловой диффузии $\Delta<\delta$. В этом случае длительность импульса свечения близка к длительности лазерного импульса, и влияние теплопроводности материала на кинетику свечения оказывается достаточно слабым (ожидаемые изменения длительности импульса свечения составляют единицы наносекунд). При условии $\Delta<\delta$ объяснить сам факт существования медленной $\left(\tau_{2} \sim 100 \mathrm{~ns}\right)$ компоненты затухания свечения не удается.

В настоящей работе рассматривается ситуация, когда глубина проникновения лазерного излучения в материал существенно превышает длину тепловой диффузии $\Delta>\delta$. В этом случае за время действия лазерного импульса температурная волна не успевает существенно выйти за пределы области тепловыделения, и соответствующее компьютерное моделирование показывает, что в затухании свечения присутствует медленная компонента с характерным временем $\tau_{2}$ порядка $100 \mathrm{~ns}$, что согласуется с результатами измерений.

Что касается возможности реализации соотношения $\Delta>\delta$ в углеродных материалах, можно принять во внимание следующие соображения. Во-первых, особенностью углеродных материалов является широкий спектр 
значений коэффициента теплопроводности: углерод может быть как хорошим теплоизолятором, так и проводником тепла [43-46]. Во-вторых, в рассматриваемых экспериментах с лазерным возбуждением теплового излучения основные процессы происходят в тонком приповерхностном слое материала, а в таких слоях обычно имеется большое количество дефектов и примесей, которые могут существенно снизить теплопроводность. В-третьих, как показано в [38], при лазерном облучении шероховатые поверхности нагреваются существенно неоднородно: даже если лазерный пучок имеет однородное распределение интенсивности по сечению, локальная температура выступов и впадин на шероховатой поверхности может отличаться на сотни градусов. Наконец, имеющиеся в литературе справочные данные по коэффициентам поглощения и теплопроводности углеродных материалов демонстрируют значительный разброс значений [47-50].

Принимая во внимание вышеперечисленные обстоятельства, представляется целесообразным провести дальнейший анализ для широкого диапазона изменения величин $\Delta$ и $\delta$.

На рис. 5 представлены рассчитанные осциллограммы свечения при возбуждении прямоугольными лазерными импульсами для различных соотношений $\Delta>\delta$ в пределах от 1 до 10. Похожие результаты получаются и для гауссовых лазерных импульсов. Для всех осциллограмм, приведенных на рис. 5, величина лазерного возбуждения подбиралась так, чтобы максимальное значение температуры составляло $3500 \mathrm{~K}$.

Как видно из рис. 5, в полулогарифмическом масштабе кривые затухания свечения заметно искривлены, что дополнительно свидетельствует в пользу сделанного в настоящей работе выбора выражения (1) для их аппроксимации. Результаты аппроксимации рассчитанных кривых затухания свечения выражением (1) приведены на рис. 6 как для случая прямоугольного, так и гауссова лазерного импульса. Как видно из рис. 6 , при $\Delta / \delta>5$ рассчитанные значения $\tau_{2}$ согласуются с измеренными значениями, приведенными на рис. 4, $a$.

Что касается механизма формирования кривых затухания теплового излучения при лазерном нагреве поверхностных слоев, следующие соображения представляются нам важными. На стадии затухания свечения (когда лазерный импульс уже закончился, т.е. $F=0$ ) уравнение (2) содержит параметр $D$ и не содержит параметр $\alpha$, однако это не означает, что кинетика затухания температуры (а также и свечения) определяется исключительно параметром $D$ и не зависит от параметра $\alpha$. Рассматриваемая кинетика затухания температуры (и свечения) зависит как от значения параметра $D$, так и от распределения температуры под поверхностью в момент начала затухания (в момент окончания лазерного импульса), а это начальное распределение температуры формируется в предшествующие моменты времени в течение действия лазерного импульса, когда $F \neq 0$ и когда в уравнение (2) входит слагаемое теплового источника, содержащее параметр $\alpha$.

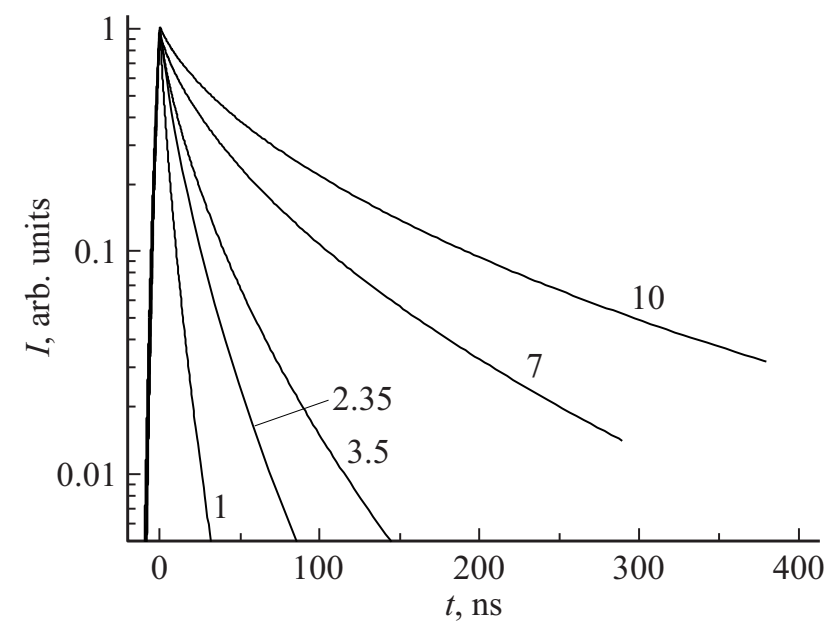

Рис. 5. Рассчитанные нормированные осциллограммы теплового излучения при возбуждении прямоугольными лазерными импульсами. Цифры возле кривых - значения $\Delta / \delta$.

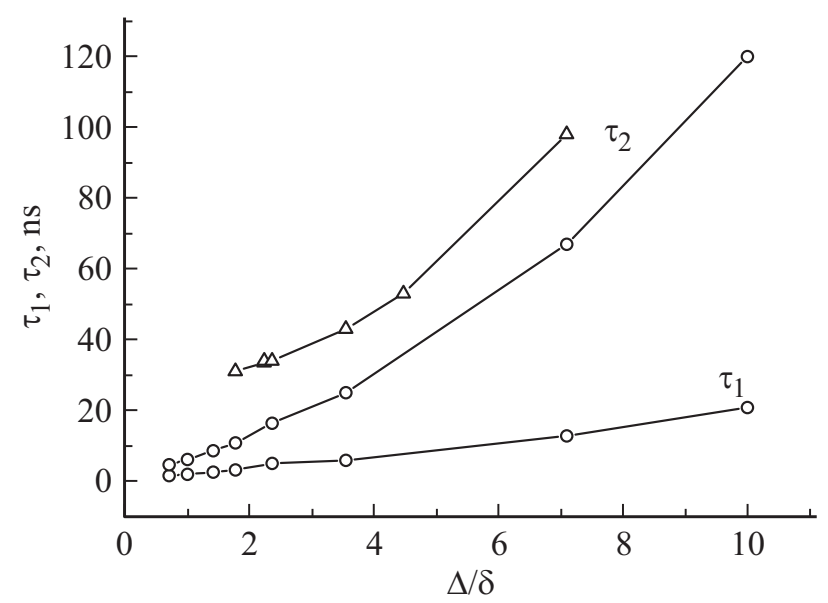

Рис. 6. Рассчитанные значения $\tau_{1}$ и $\tau_{2}$ как функции $\Delta / \delta$ при возбуждении прямоугольными (кружки) и гауссовыми (треугольники) лазерными импульсами.

Как видно из рис. 6 , рассчитанные значения $\tau_{2}$ для прямоугольного и гауссова лазерных импульсов заметно отличаются, что вполне соответствует приведенным выше соображениям. Поскольку использованные в настоящей работе импульсы лазера с модуляцией добротности имели колоколообразную форму, в дальнейшем мы используем для анализа результаты, приведенные на рис. 6 для гауссова лазерного импульса.

Полученные в настоящей работе результаты моделирования дают основания сделать вывод, что величина $\tau_{2}$ определяется отношением $\Delta / \delta$, а не только величиной коэффициента температуропроводности $D$. Как свидетельствуют результаты расчетов, приведенные в таблице, при варьировании величин $\Delta$ и $\delta$ с сохранением постоянства их отношения $\Delta / \delta$ рассчитанные величины $\tau_{2}$ также остаются постоянными.

Рассмотренная выше особенность затухания свечения (зависимость $\tau_{2}$ от отношения $\Delta / \delta$ ) дает возможность 
Рассчитанные значения $\tau_{2}$ для разных значений $\Delta$ и $\delta$

\begin{tabular}{c|c|c|c|c|c|c|c}
\hline $\begin{array}{c}\kappa, \\
\mathrm{Wm}^{-1} \mathrm{~K}^{-1}\end{array}$ & $\begin{array}{c}C_{p}, \\
\mathrm{Jm}^{-3} \mathrm{~K}^{-1}\end{array}$ & $\begin{array}{c}D, \\
\mathrm{~m}^{2} \mathrm{~s}^{-1}\end{array}$ & $\begin{array}{c}\delta, \\
\mathrm{nm}\end{array}$ & $\begin{array}{c}\alpha, \\
10^{6} \mathrm{~m}^{-1}\end{array}$ & $\begin{array}{c}\Delta, \\
\mathrm{nm}\end{array}$ & $\Delta / \delta$ & $\begin{array}{c}\tau_{2}, \\
\mathrm{~ns}\end{array}$ \\
\hline 1 & $10^{6}$ & $10^{-6}$ & 141.4 & 1.414 & 707 & 5 & 43.3 \\
1 & $10^{5}$ & $10^{-5}$ & 447.2 & 0.447 & 2236 & 5 & 43.7 \\
10 & $10^{6}$ & $10^{-5}$ & 447.2 & 0.447 & 2236 & 5 & 43.5
\end{tabular}

оценить величину коэффициента температуропроводности поверхностного слоя облучаемого материала с использованием измеренного значения $\tau_{2}$ и справочного значения $\alpha$. Например, для $\tau_{2}=100 \mathrm{~ns}$ из рис. 6 получаем $\Delta / \delta \approx 7$, и с использованием справочных данных $[48,49] \alpha=10^{7} \mathrm{~m}^{-1}$ получаем оценку коэффициента температуропроводности $D \approx 10^{-8} \mathrm{~m}^{2} \cdot \mathrm{s}^{-1}$. Используя полученную оценку $D$, можно оценить коэффициент теплопроводности слоя. Предполагая, что теплоемкость при высокой температуре достигает значений около $2000 \mathrm{~J} \cdot \mathrm{kg}^{-1} \cdot \mathrm{K}^{-1}$, а плотность порядка $2000 \mathrm{~kg} \cdot \mathrm{m}^{-3}$, получаем оценку коэффициента теплопроводности $\kappa \approx 0.04 \mathrm{~W} \cdot \mathrm{m}^{-1} \cdot \mathrm{K}^{-1}$, что по порядку величины соответствует теплоизоляционным материалам [46]. Столь небольшое значение коэффициента теплопроводности может быть связано с зернистой или чешуйчатой структурой поверхностных слоев использованных в настоящей работе образцов. Кроме того, возможно, сыграли свою роль температурные зависимости коэффициента теплопроводности и теплоемкости углерода [45,51].

В конце следует дополнительно подчеркнуть, что предложенная процедура дает оценку коэффициента температуропроводности, во-первых, для материала, содержащегося в тонком приповерхностном слое, и, вовторых, при высокой температуре (при которой наблюдается свечение). Последнее обстоятельство может оказаться важным, поскольку измерения тепловых характеристик при высоких температурах традиционными методами весьма затруднительны.

\section{Заключение}

Поскольку настоящая работа представляет собой первую попытку анализа особенностей кривых затухания свечения тонких поверхностных слоев материалов при импульсном лазерном нагреве до высоких температур, полученные результаты проливают свет на наиболее простые закономерности исследуемых процессов и формулируют вопросы для дальнейших исследований. Что касается точности предложенного метода оценки температуропроводности в поверхностном слое материала при высокой температуре, отметим следующее. В настоящей работе при компьютерном моделировании не учитывались температурные зависимости параметров материала, что, несомненно, снижает точность расчетов в широком температурном интервале. Кроме того, в соответствии с выводами, сделанными в работах [35,38] о роли шероховатости поверхности в процессах лазерного нагрева поверхностных слоев материалов, в формировании кривой затухания свечения при импульсном лазерном возбуждении шероховатость облучаемой поверхности может стать одним из определяющих факторов, однако рассмотрение этого вопроса выходит за рамки данной работы и требует отдельного исследования.

\section{Финансирование работы}

Данная работа была частично поддержана грантом № 2006 (2018 г.) Института электроники Университета Шизуоки, а также проектами 16БФ051-01 и 19БФ051-02 Киевского национального университета имени Тараса Шевченко.

\section{Конфликт интересов}

Авторы заявляют, что у них нет конфликта интересов.

\section{Список литературы}

[1] Жаров В.П., Летохов В.С. Лазерная оптико-акустическая спектроскопия. М.: Наука, 1984. 320 с.; Zharov V.P., Letokhov V.S. Laser Optoacoustical Spectroscopy (Springer Series in Optical Sciences. V. 37). Berlin: Springer, 1986. $327 \mathrm{p}$.

[2] Mandelis A. // Physics Today. 2000. V. 53. N 8. P. 29.

[3] Lin L.T., Archibald D.D., Honigs D.E. // Appl.Spectr. 1988. V. 42. N 3. P. 477.

[4] Tsuge A., Uwamino Y., Ishizuka T. // Appl. Spectr. 1989. V. 43. N 7. P. 1145.

[5] Galan-Freyle N.J., Pacheco-Londono L.C., Figueroa-Navedo A.M., Hernandez-Rivera S.P. // Appl. Spectr. 2015. V. 69. N 5. P. 535.

[6] Mandelis A. // Solid-State Electronics. 1998. V. 42. N 1. P. 1.

[7] Mandelis A., Munidasa M. // Int J. Thermophys. 1994. V. 15. N 6. P. 1299.

[8] Park H.K., Grigoropoulos C.P., Tam A.C. // Int. J. Thermophys. 1995. V. 16. N 4. P. 973.

[9] Fuente R., Mendioroz A., Apinaniz E., Salazar A. // Int. J. Thermophys. 2012. V. 33. P. 1876.

[10] Fleurence N., Hay B., Davee G., Cappella A., Foulon E. // Phys. Stat. Sol. A, 2015. V. 212. N 3. P. 535.

[11] Pham Tu Quoc S., Cheymol G., Semerok A. // Rev. Sci. Instr. 2014. V. 85. P. 054903.

[12] Kruse D., Prekel H., Goch G., Walther H.G. // Proc. Estonian Acad. Sci. Eng. 2007. V. 13. N 4. P. 423.

[13] Chen G., Borca-Tasciuc T. // Int. J. Heat Mass Transfer. 1998. V. 41. N 15. P. 2279.

[14] Markham J.R., Best P.E., Solomon P.R. // Appl. Spectr. 1994. V. 48. N 2. P. 265.

[15] Loarer T., Greffet J.-J., Huetz-Aubert M. // Appl. Opt. 1990. V. 29. N 7. P. 979.

[16] Vander Wal R.L. // Appl. Phys. B. 2009. V. 96. P. 601.

[17] Schulz C., Kock B.F., Hofmann M. et. al. // Appl. Phys. B. 2006. V. 83. P. 333.

[18] Michelsen H.A., Liu F., Kock B.F., et. al. // Appl. Phys. B. 2007. V. 87. P. 503.

[19] Sapmaz H., Lin C.-X., Ghenai C. // Exp. Fluids. 2008. V. 44. P. 137. 
[20] Michelsen H.A. // J. Chem. Phys. 2003. V. 118. P. 7012.

[21] Goulay F., Schrader P.E., Michelsen H.A. // Appl. Phys. B. 2009. V. 96. P. 613.

[22] Charwathr M., Suntz H., Buckhorn H. // Appl. Phys. B. 2006. V. 83. P. 435.

[23] Zelensky S. // J. Opt. A: Pure Appl. Opt. 1999. V. 1. P. 454.

[24] Zelensky S. // J. Lumin. 2003. V. 104. N 1-2. P. 27.

[25] Rulik Ju.Ju., Mikhailenko N.M., Zelensky S.E., Kolesnik A.S. // Semicond. Phys. Quant. Electron. Optoelectron. 2007. V. 10. N 2. P. 6.

[26] Zelensky S. // J. Phys.: Cond. Mat. 1998. V. 10. N 32. P. 7267.

[27] Kopyshinsky A.V., Lazorenko Ya.P., Zelensky S.E. // Functional Materials. 2011. V. 18. N 1. P. 116.

[28] Zelensky S.E., Kolesnik A.S., Kopyshinsky A.V., et. al. // Ukrainian J. Phys. 2009. V. 54. N 10. P. 983.

[29] Zelenska K.S., Poperenko L.V., Kanev K., et. al. // Opt. Laser Techn. 2016. V. 76. P. 96.

[30] Mansour K., Soileau M.J., Van Stryland E.W. // J. Opt. Soc. Am. B. 1992. V. 9. N 7. P. 1100.

[31] Vivien L., Lancon P., Riehl D. et al. // Carbon. 2002. V. 40. P. 1789.

[32] Zelensky S. // Semicond. Phys. Quant. Electron. Optoelectron. 2004. V. 7. N 2. P. 190.

[33] Chin K.C., Gohel A., Chen W.Z. et al. // Chem. Phys. Lett. 2005. V. 409. P. 85.

[34] Videnichev D.A., Belousova I.M. // Appl. Phys. B: Lasers and Optics. 2014. V. 115. P. 401.

[35] Zelensky S.E., Poperenko L.V., Kopyshinsky A.V., Zelenska K.S. // Proc. SPIE. 8434. Nonlinear Optics and Applications. VI. 2012. 84341H.

[36] Kopyshinsky A.V., Zelensky S.E., Gomon E.A., Rozouvan S.G., Kolesnik A.S. // Semicond. Phys. Quant. Electron. Optoelectron. 2012. V. 15. N 4. P. 376.

[37] Zelensky S.E., Zelenska K.S. // Proc. SPIE 8772. Nonlinear Optics and Applications VII. 2013. 87721P.

[38] Zelenska K.S., Zelensky S.E., Kopyshinsky A.V., Rozouvan S.G., Aoki T. // Japan. J. Appl. Phys.: Conf. Proc. 2016. V. 4. P. $011106-1-6$.

[39] Zelenska K., Zelensky S., Kopyshinsky A. // Thai J. Nanosci. Nanotechnol. 2017. V. 2. N 1. P. 1.

[40] Karpovych V., Zelenska K., Yablochkov S., Zelensky S., Aoki T. // Thai J. Nanosci. Nanotechnol. 2017. V. 2. N 2. P. 14.

[41] Kokhan M., Koleshnia I., Zelensky S., Hayakawa Y., Aoki T. // Opt. Laser Techn. 2018. V. 108. P. 150.

[42] Kokhan M., Koleshnia I., Zelensky S., Aoki T. // Proc. SPIE 10097. High-Power Laser Materials Processing: Applications, Diagnostics, and Systems VI. 2017. 100970G.

[43] Grujicic M., Zhao C.L., Biggers S.B., Kennedy J.M., Morgan D.R. // Proc. IMechE L: J. Mater. Design and Appl. 2005. V. 219. N 4. P. 217.

[44] Kutuzov S.V., Vasil'chenko G.N., Chirka T.V., Panov E.N. // Refract. Industr. Ceram. 2013. V. 54. N 1. P. 39.

[45] Pierson H.O. Handbook of Carbon, Graphite, Diamond and Fullerenes: Processing, Properties and Applications. Park Ridge, New Jersey: Noyes Publications, 1993.

[46] International Critical Tables of Numerical Data, Physics, Chemistry and Technology. / Ed. by Washburn E.W. Knovel, Norwich, NY, 2003.

[47] Yon J., Lemaire R., Therssen E., et. al. // Appl. Phys. B. 2011. V. 104. P. 253.

[48] Djurisic A.B., Li E.H. // J. Appl. Phys. 1999. V. 85. P. 7404.
[49] Querry M.R. Contractor Report CRDC-CR-85034. 1985.

[50] Papoular R.J., Papoular R. // Month. Not. Roy. Astr. Soc. 2014. V. 443. N 4. P. 2974.

[51] Ho C.Y., Powell R.W., Liley P.E. // J. Phys. Chem. Ref. Data. 1974. V. 3 (Suppl. 1). P. 1-756. 